\title{
Introduction. Pour un inventaire des collections océaniennes en France : regard rétrospectif en 2021
}

Introduction. For an inventory of Oceanic collections in French museums. A retrospective regard in 2021

Magali Mélandri et Hélène Guiot

\section{(2) OpenEdition}

\section{Journals}

Édition électronique

URL : https://journals.openedition.org/jso/13023

DOI : $10.4000 /$ jso. 13023

ISBN : $1760-7256$

ISSN : $1760-7256$

Éditeur

Société des océanistes

Édition imprimée

Date de publication : 30 juin 2021

Pagination : 5-20

ISBN : 978-2-85430-140-3

ISSN : 0300-953x

Référence électronique

Magali Mélandri et Hélène Guiot, «Introduction. Pour un inventaire des collections océaniennes en France : regard rétrospectif en 2021 », Journal de la Société des Océanistes [En ligne], 152 | 2021, mis en ligne le 30 juin 2021, consulté le 03 janvier 2023. URL : http://journals.openedition.org/jso/13023 ; DOI : https://doi.org/10.4000/jso.13023

\section{(c) $($ ) $(9)$}

Creative Commons - Attribution - Pas d'Utilisation Commerciale - Pas de Modification 4.0 International - CC BY-NC-ND 4.0

https://creativecommons.org/licenses/by-nc-nd/4.0/ 


\title{
Introduction. Pour un inventaire des collections océaniennes en France : regard rétrospectif en 2021
}

par

\author{
Hélène GUIOT* et Magali MÉLANDRI**
}

En décembre 1945, paraissait le premier tome du Journal de la société des Océanistes. Au sommaire, figurait un article de Marie-Charlotte Laroche - alors attachée au département Océanie du musée de l'Homme et l'une des fondatrices de la Société des Océanistes - intitulé : "Pour un inventaire des collections océaniennes en France ». Par la suite, plus d'une centaine d'articles furent publiés dans cette même revue, qui traitaient des objets océaniens, qu'il s'agisse de ceux conservés au musée de l'Homme (Girard, 1945, 1953), dans les musées de région, ou encore de ceux réunis par des approches spécifiques comme dans les numéros plus récents (Leblic, 2013 ; Kaufmann, Peltier et Schindlbeck, 2018). Soulignons également les nombreux comptes rendus de catalogues d'exposition dont la revue se fait régulièrement l'écho.

Ainsi, dès ses débuts, le Journal de la société des Océanistes a donné à comprendre les différentes approches, méthodologiques et thématiques, développées en France autour des collections et des objets océaniens depuis le milieu du Xx siècle, sans pour autant qu'un dossier thématique dédié n'y soit pleinement consacré. En 2019, sous l'impulsion d'Emmanuel Kasarhérou, alors président de la Société des Océanistes, naît l'idée de tenter l'exercice. Fortes de récentes avancées en matière d'inventaire et de préoccupations de plus en plus pressantes sur les questions de provenances, sous-tendues par les problématiques de restitution des objets extra-occidentaux, les propositions d'articles ont afflué. Indice d'une prise de conscience par le monde muséal et académique du nécessaire travail d'approfondissement des connaissances en matière de biographies des objets, cette affluence témoigne aussi de sa mise en œuvre effective et efficace.

On recense 65000 objets océaniens environ, répartis dans cent seize musées ${ }^{1}$ à travers le territoire français, entendu dans sa territorialité européenne et outre-mer. La tâche s'avère donc considérable pour embrasser leur étude, leurs interrelations au sein de ces institutions et au-delà, ou encore leurs points de contact avec d'autres collections extra-européennes. S'ajoute à ce vertige du chiffre, celui d'une interdisciplinarité maximale, inévitablement convoquée lorsqu'on s'attache à traiter du lien entre objets et musées : ethnologie de l'Océanie, anthropologie des techniques, anthropologie de l'art, histoire des arts d'Océanie, histoire des explorations et des voyages, histoire des sciences, histoire des collectes et des collections, histoire des institutions, histoire de la muséographie, histoire religieuse, politique et économique, etc., chacune de ces disciplines portant vis-à-vis des objets des discours et des régimes de valeurs propres.

Considérée comme un "fait anthropologique " (Pomian, 2008 : 5), une collection dans sa définition universelle est

« [un] ensemble d'objets naturels ou artificiels extraits du circuit d'activités utilitaires soumis à une protection spéciale et exposés au regard dans un lieu clos destiné à cet effet " (Pomian, 2021 : 10)

Son étude, en ce qu'elle permet

«d'appréhender les attitudes cognitives et esthétiques, les pratiques intellectuelles, les relations sociales, les représentations " (Pomian, 2008: 5), implique, dans

1. Un travail de mise à jour de ces chiffres, établis en 2007 grâce au travail mené par Roger Boulay (2007), reste aujourd'hui à réaliser, suite aux récolements et inventaires engagés par les musées ou via des projets de recherche transversaux.

*Ethno-archéologue, chargée de cours INALCO ASEP, helenguiot@gmail.com

** Directrice déléguée à la conservation, musée départemental Albert-Kahn, Boulogne-Billancourt, mmelandri@hauts-de-seine.fr. 
le cas des collections océaniennes, une façon de restituer, en démêlant d'inévitables nœuds biographiques, le parcours des objets depuis leur création jusqu’à leur(s) réception(s). Sans cette enquête biographique, un objet mal on non identifié devient muet et perd une grande part de son intérêt en matière de récit, de médiation. Mission du chercheur, du professionnel de musée ${ }^{2}$, cette démarche réflexive s'est tardivement ouverte, intégrant désormais les voix des communautés culturelles d'où proviennent les objets, mettant davantage en avant leurs savoirs et leurs savoir-faire dans une perspective croisée et critique.

D'un point de vue méthodologique, l'étude des collections muséales océaniennes - mais l'on pourrait l'étendre à l'ensemble des collections extra-européennes - s'appuie sur une étude matérielle des objets au sein d'une collection donnée: caractéristiques physiques et techniques (endogènes et exogènes), marquages et étiquettes, traces d'usages d'origine et postérieures (par exemple application de cires, résidus de peinture et traces d'oxydations issus des présentations muséographiques). A cette étude par l'œil - augmentée aujourd'hui d'une panoplie d'équipements scientifiques de pointe (voir LeclercCaffarel et Servain-Riviale, ce volume, pp. 155-168) -, s'ajoute l'analyse documentaire : archives et documentation ${ }^{3}$ propres à l'institution et à celles qui ont pu la précéder ; archives municipales, départementales, militaires, missionnaires, diplomatiques etc.; documentation iconographique (dessins, gravures, photographies, films) liée à l'objet et à ses différents contextes biographiques et muséographiques ; bibliographie spécialisée selon la provenance géographique et culturelle des œuvres (voir ce volume, Chataigner, pp. 127-136 ; Le Cornec, pp. 105-114).

Cette recherche n'est jamais isolée et amène régulièrement le croisement de ces (res)sources, avec celles d'autres collections ou/et d'autres musées. Dans une même région, les provenances communes de collections aujourd'hui conservées dans des musées distincts ne sont pas rares et peuvent éclairer mutuellement les recherches ${ }^{4}$. De même, l'étude comparée d'un même corpus typologique ou géographique d'objets présent dans différentes institutions participe d'une histoire de l'art encore parcellaire pour le monde océanien. L'entrée par collecteurs/donateurs/vendeurs permet aussi le ricochet d'une institution à l'autre et la reconstitution de collectes à entrées multiples ( $c f$. par exemple Jacquemin, 1998 : 25, Lavondès, 1990 : 28-
32 et Leclerc-Caffarel, 2008 pour les collections liées aux voyages de Jules Sébastien Dumont D'Urville).

Cette combinatoire, tant disciplinaire que méthodologique, et sa richesse de positionnements, sont essentiels pour mener le travail d'analyse et de décryptage du processus de formation des collections, afin d'en dresser le récit - même chaotique - et de le partager.

\section{Premiers voyages : de l'objet collecté aux musées}

La collecte d'objets d'Océanie et leur réception relèvent d'une variété d'époques, de perceptions de l'altérité et du monde, d'une multiplicité d'acteurs entre statut officiel et rôle personnel - et de situations de rencontres. In situ, ces artefacts ont pu être échangés, subtilisés, achetés, prélevés tels des échantillons, ou encore reçus en cadeau. L'ensemble des voyages menés dans le Pacifique depuis le Xvi siècle a établi des points de contact et tracé des routes diverses qui ont ensuite façonné la construction de collections au sein des musées.

\section{Objet de curiosité et de richesse}

Magellan (1519-1522) et les Espagnols (Balboa en 1513, Legazpi 1564-1565, Mendaña 1567-1569 et 1595-1596, Quiros en 1595-1596 puis 1605-1606, cf. par exemple Baert, 1999) inaugurent les voyages d'exploration de l'océan Pacifique, suivis par les Hollandais (Willem Janszoon, 1606; Schouten et Le Maire, 1615-1616 ; Abel Janszoon Tasman, 16421643). Dès lors, une image construite de l'Océanie et de ses habitants commence à circuler : aux motivations économiques, politiques et en partie évangélisatrices, s'associent la recherche d'un continent austral, mais aussi le sentiment d'avoir découvert une terre d'abondance et un Paradis terrestre (Baert, 1999 ; Bonnemaison, 1986 : 15-39). La révélation des richesses du monde, qui paraissent infinies, suscite le développement des cabinets de curiosités à travers l'Europe. Dans une tentative de connaissance encyclopédique "tout ce qui vient de loin, dans le temps comme dans l'espace, est désirable » (Schnapper, 1997 : 21). Les productions humaines trouvent alors grâce aux yeux de ces curieux et amateurs érudits (princes et rois, mais aussi médecins ou pharmaciens, etc.), comme reflets de l'incroyable diversité du monde et de l'inépuisable capacité d'invention de Dieu.

2. Celles-ci sont clairement énoncées dans le code du patrimoine de 2004 (IV, IV, 1, art. L441-2) : conserver, restaurer, étudier et enrichir leurs collections; rendre leurs collections accessibles au public le plus large ; concevoir et mettre en ouvre des actions d'éducation et de diffusion visant à assurer l'égal accès de tous à la culture ; contribuer aux progrès de la connaissance et de la recherche ainsi qu’à leur diffusion.

3. Pour quelques exemples : documents administratifs liés aux modalités d'entrée dans les collections du musée, inventaires anciens et contemporains, rapports de mission de collecte, correspondances administratives et scientifiques diverses, dossiers d'œuvres, rapports de restauration, chronologie des expositions in situ et prêts extérieurs, etc.

4. L'étude approfondie menée par le muséum d'Histoire naturelle de Toulouse sur les frères Savès (voir Bonvin, Dufau et Granier dans ce volume, pp. 49-60), pourrait par exemple bénéficier au musée d’Aquitaine dont environ cinquante pièces ont été acquises, d'après les inventaires anciens, auprès de ces mêmes marchands toulousains. 
L'objet créé et utilisé au sein d'une société d'Océanie devient artificialia des mers du Sud. Ainsi, apparaissent en France, comme ailleurs en Europe, des artefacts océaniens,

"dans les cabinets des amateurs, voisinant, en un curieux mélange, les coquillages, les pierres précieuses et les étrangetés de la nature; mais en définitive, ils échouent souvent entre les mains de collectionneurs, célèbres ou anonymes. » (Jacquemin, $1991: 14$ )

Leur présence est signalée surtout vers la fin du XVIII ${ }^{\mathrm{e}}$ siècle, comme l'attestent les cabinets du comte Leroy de Barde (Boulay, 1997 : 33-35) ou celui du baron Vivant Denon (Jacquemin, 1999).

\section{Objet témoin d'explorations scientifiques}

Durant ce même XvIII ${ }^{e}$ siècle, celui des Lumières, savants et lettrés s'interrogent sur la nature de l'Homme, et l'exploration des mers s'organise de manière systémique. Les navigateurs étant tenus de rapporter des preuves de leurs découvertes, l'objet exotique endosse ce rôle, enrichi des gravures ou dessins les mettant en situation. Support d'un discours tourné vers une forme de connaissance encyclopédique, il atteste l'existence des contrées visitées et nouvellement cartographiées ainsi que des populations rencontrées.

La première expédition française dans le Pacifique, menée par Louis-Antoine de Bougainville (17661769), est destinée à assurer la maîtrise de nouvelles voies maritimes et à conquérir des terres inconnues au nom du roi. Ce voyage, qui associera pour longtemps le mythe édénique aux îles de l'Océanie, engage la France dans ce vaste champ d'exploration que devient alors l'océan Pacifique. S'ensuivent les expéditions de Jean-François-Marie de Surville (17681769), Marc-Joseph Marion du Fresne (1770-1773), Jean-François de La Pérouse (1785-1788) et JosephAntoine Bruni d'Entrecasteaux (1791-1794). Pour remplir les visées scientifiques de ces explorations (cartographie, collectes d'échantillons, descriptions des populations rencontrées), les équipages incluent des savants issus de la société civile ${ }^{5}$.

Puis, au $\mathrm{XIX}^{\mathrm{e}}$ siècle, les voyages de circumnavigation (voir par exemple Blais, 2000), tels ceux de Louis-Claude de Saulces de Freycinet (1817-1820), Louis-Isidore Duperrey (1822-1825) et Jules-Sébastien-César Dumont d'Urville (1826-1829 et 18371840), emploient surtout des scientifiques issus du monde militaire. Ces expéditions doivent également revenir munies de toutes les informations commerciales et politiques susceptibles d'être utiles à une conquête territoriale. Les objets sont alors collectés parmi des centaines d'autres productions naturelles, comme autant de spécimens d'une histoire naturelle du monde. Malgré tout, ces objets visent à

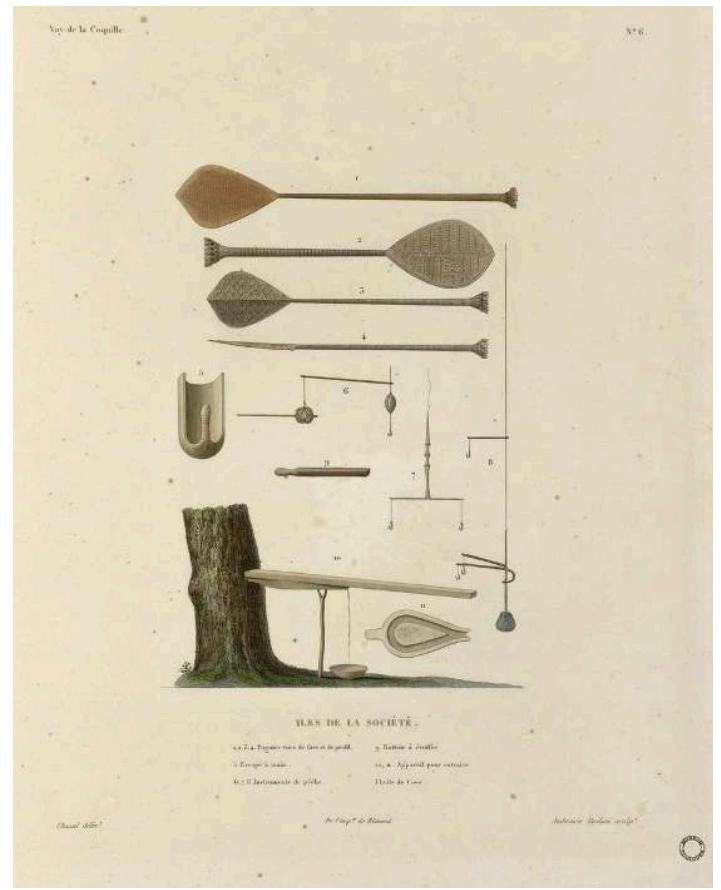

Document 1. - Planche 6 : îles de la Société (Duperrey, 1825-1830) (Muséum d'Histoire naturelle de Toulouse, ville de Toulouse, établissement de conservation-Rosalis)

" éclaircir les mours et les usages des nations et des peuplades non connues » (Edmé-François Jomard, 1831, cité par Hamy, 1880, in Grognet, 2005 : 51)

\section{Un regard ethnographique prend naissance}

\section{Objet des colonies, matériel d'étude et preuves de conversion}

Durant la seconde moitié du XIX ${ }^{e}$ siècle, la variété des formes de voyages contribue à la diversité des intérêts portés sur les objets d'ailleurs. À partir de 1840, l'objectif colonial français s'affirme avec la mise sous protectorat des îles Marquises, en 1842, par Abel Aubert du Petit-Thouars ou Dupetit-Thouars. Un nouveau public collecte et documente les productions matérielles insulaires : le personnel de l'administration coloniale, composé de militaires et de fonctionnaires, les colons eux-mêmes, ainsi que les missionnaires dont l'implantation coïncide avec le mouvement colonial. Pour ces derniers, les objets collectés constituent les preuves concrètes de leur action évangélisatrice : les Océaniens, renonçant à leurs cultes, se défont des réceptacles divins et d'autres supports des rituels de leur religion, désormais considérée comme païenne. Certains les dérobent à la vue des missionnaires et adaptent leurs pratiques traditionnelles.

Les nouveaux résidents sont parfois en lien avec les sociétés savantes qui, prenant leur essor,

« sollicitent voyageurs et résidents afin qu' ils leur fournissent un matériel d'étude » (Patole-Edoumba, 2009 : 176)

5. Comme Philibert Commerson (1727-1773), médecin et naturaliste de l'expédition de Bougainville. 


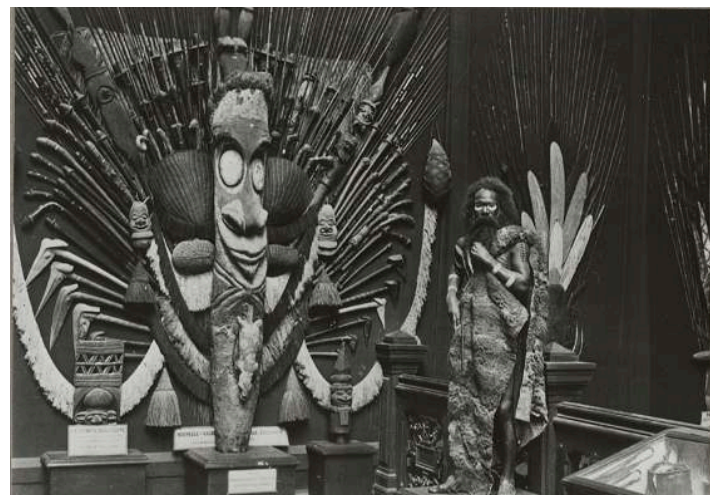

Рното 1. - Objets océaniens au musée d'ethnographie du Trocadéro, 1895 (anonyme, PP0001442, MQB-JC)

Tout au long du XIX ${ }^{e}$ siècle, la naissance de nouvelles structures muséales est en marche, nourrie par le versement des collections exotiques issues des cabinets privés des collectionneurs et des collections royales du siècle précédent, des collectes des premiers voyageurs et explorateurs, ainsi que par les objets présentés dans le cadre des expositions universelles ${ }^{6}$ puis coloniales à travers le pays (Jacquemin, 1991). L'émergence de la discipline anthropologique dans la seconde moitié du siècle, intrinsèquement liée à l'histoire des premiers musées d'ethnographie, voit la multiplication des missions de terrain qui viendront nourrir les réserves et les vitrines de ces musées (Dias, 1991 ; Delpuech, 2017). Les collections sont alors en mouvement perpétuel (échanges, dépôts, transferts...), prises dans une succession de courants de pensées, de choix scientifiques et politiques, qui n'ont cessé d'opérer des mécanismes de sélection et d'interprétations de ces ouvres au sein des musées. L'expansion des schémas évolutionnistes puis diffusionnistes et le développement en parallèle d'une archéologie comparée contribuent à semer une confusion, fort perceptible dans la muséographie des premiers musées des sciences de l'homme : bien qu'exposés, les objets peinent à retrouver leur identité, souvent mélangés à d'autres corpus ou confondus avec d'autres provenances.

\section{Objet d'art, objet de musées}

À la fin du XIX ${ }^{e}$ siècle, les voyages vers le lointain océan Pacifique donnent naissance à une nouvelle forme de littérature qui inspire les artistes ${ }^{7}$. Au tournant $\mathrm{du} \mathrm{Xx}^{\mathrm{e}}$ siècle, le groupe des "avant-gardistes"

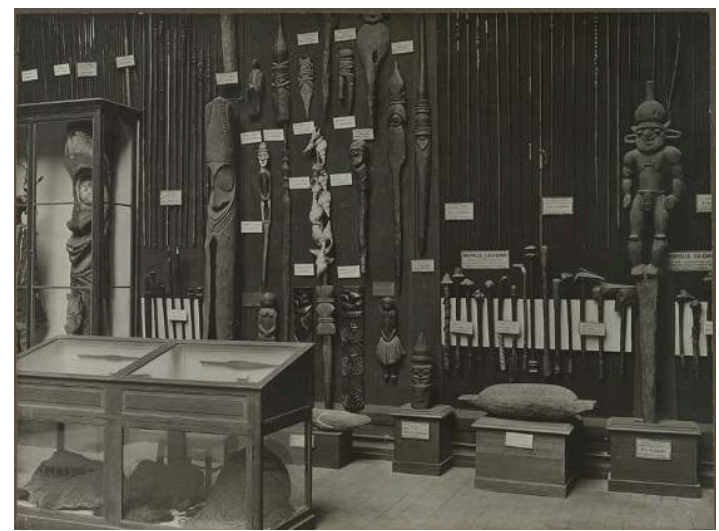

Рното 2. - Objets océaniens, vue prise dans une salle du musée d'ethnographie du Trocadéro, 1930 (anonyme, PP0001267, MQB-JC)

découvre des objets en provenance d'Afrique, des Amériques et d'Océanie. Ces artefacts exotiques deviennent des objets d'art, miroirs de leurs préoccupations esthétiques (Peltier, 1979 et 1984). Dans ce mouvement de rapprochement avec l'art occidental, où s'associent critiques d'art, marchands, collectionneurs et écrivains, Apollinaire appelle en 1909 à l'introduction de ces œuvres au Louvre (Apollinaire, 1991 [1909] : 122-124).

Durant les $\mathrm{Xx}^{\mathrm{e}}$ et $\mathrm{XXI}^{\mathrm{e}}$ siècles, les pratiques de collecte n'ont de cesse de se diversifier. À celles des collections occasionnelles des voyageurs et des amateurs éclairés viennent s'ajouter les collectes des expéditions qui se placent sous l'égide d'institutions muséales, et s'effectuent dans un contexte scientifique naissant ${ }^{8}$. Les matériaux accumulés constituent des témoignages inestimables des manières de faire océaniennes et de leurs hybridations contemporaines. Lordonnancement scientifique qui se fait jour dans les vitrines des musées redonne visibilité aux objets, tandis que les modalités de présentation des collections s'enrichissent progressivement de nouvelles perspectives, tournées à la fois vers une appréciation tant scientifique qu'esthétique des œuvres.

Les lieux de réception des objets extra-européens illustrent cette pluralité. En parallèle des institutions nationales ${ }^{9}$ - chronologiquement, le musée de Marine du Louvre, le musée d'Archéologie nationale, celui de l'Artillerie, le musée d'Ethnographie du Trocadéro (MET), le musée de l'Homme (MH), les musées des arts d'Afrique et d'Océanie (MNAAo) et du quai Branly-Jacques Chirac (MQB-JC) - une constellation de musées à travers la France opère selon des schémas

6. En 1878, le mécène Raphaël Bischoffsheim fait don, au musée d'Ethnographie du Trocadéro (MET) nouvellement créé, d'une centaine d'artefacts aborigènes d'Australie présentés lors de l'exposition universelle. Ces objets sont aujourd'hui conservés au MQB-JC $\left(71.1880 .39^{*}\right)$

7. Tels les récits de Pierre Loti, Le mariage de Loti-Rarahu (1880), Paul Gauguin avec Noa Noa (1901) ou Victor Ségalen et Les Immémoriaux (1907), de même que ceux de Herman Melville et de Robert Louis Stevenson.

8. Parmi les collectes institutionnelles effectuées, citons pour le MET : mission Collardo en Mélanésie (1925), O’Reilly à Bougainville (1934), Métraux-Lavachery à l'île de Pâques (1934-1935), Leenhardt en Nouvelle-Calédonie (1938-1939). Pour le MH et le mNAAO : Françoise Girard (Nouvelle-Guinée, 1954-1955, 1976), Daniel de Coppet (îles Salomon, années 1960), Marie-Claire Bataille (Tonga : 1974, 1985, île de Pâques), Jean Guiart (Nouvelle-Calédonie, Nouvelle-Guinée, 1962-1972), Roger Boulay (Vanuatu, Australie, 1991-1992), Christian Coiffier (Nouvelle-Guinée, années 1990-2000), Philippe Peltier (Nouvelle-Guinée 1992, Australie, années 2000) qui collecte ensuite pour le MQB-JC, tout comme Magali Mélandri (îles Salomon, 2014). 
d'acquisition proches de ceux des musées nationaux, auxquels vient s'ajouter l'histoire locale, individuelle et singulière. Leurs identités sont de fait multiples : muséums d'histoire naturelle, musées d'archéologie, musées coloniaux, musées d'art et d'histoire, musées municipaux, musées des civilisations, musées des techniques, musées privés d'amateurs ${ }^{10}$. La compréhension de cette complexité du paysage muséal et des trajectoires des objets au sein de ces institutions, parfois éloignées les unes des autres, sur le territoire comme dans le temps de leur création, fait partie des enjeux de l'étude des collections océaniennes.

\section{Biographies d'objets, histoires de collections, provenances : jalons historiques}

\section{Les pionniers}

On peut s'étonner de constater que les premières études de collections océaniennes conservées en France soient menées, au début $\mathrm{du} \mathrm{xx}^{\mathrm{e}}$ siècle, par des spécialistes internationaux. Leur recherche suit une orientation monographique. C'est le cas de l'allemand Karl von den Steinen qui, après une mission ethnographique de six mois aux îles Marquises (1897-1898), déploie sa recherche dans les musées des États-Unis et d'Europe afin d'achever l'étude de la culture matérielle de cet archipel.

"J'ai parcouru la plupart des pays européens sur la piste de ces objets: de Bordeaux à Saint-Pétersbourg, de Stockholm à Rome, mais avant tout en Angleterre et avec plus de succès en France, en tant que métropole de cette colonie les collections historiques de la Marine s'y trouvent, ainsi que de nombreux musées de province; de Caen à Grenoble. » (Steinen, 2006 :v)

Il visite également les institutions d'Annecy, de Boulogne-sur-Mer, Cherbourg, Colmar, Douai, Dunkerque et Lille. Son travail, composé de descriptions, de photographies et de dessins, comptant plus de mille pièces, est publié en 1925 .

Comme le soulignait Marie-Charlotte Laroche (1945 : 55), le Bernice Pauahi Bishop Museum fait preuve d'une "intelligente et féconde activité ": dès 1896, son directeur et fondateur, William T. Brigham, se rend dans les musées d'Océanie, des États-Unis et d'Europe. Il faut souligner cette démarche remarquablement innovante et pionnière dont le but est de

"visit and examine the principal Ethnological museums of the world, hoping that besides studying the rare and now unattainable ethnological objects in those museums, he might arrange exchanges of duplicates or publications, obtain photographs of interesting specimens, and learn what might be new or of value in the arrangement or management of such institutions, and in the preservation of their contents. " (Brigham, $1898: 1)$

Lors de son premier séjour en France, en 1896, Brigham se fixe à Paris,

"the home of Broca and once the chief dwelling place of Anthropology and Ethnology" (1898:37)

Il visite le musée de Marine au palais du Louvre, le jardin des Plantes et la galerie ethnographique du musée d'Artillerie (hôtel des Invalides), où il observe avec attention les pièces hawaiiennes. Lors d'un second voyage, en 1912 (Brigham, 1913), il se rend de nouveau au Jardin des Plantes, puis au MET, et part pour Marseille (où il est impressionné par l'architecture du palais Longchamp), avant de se rendre au musée océanographique de Monaco, but de sa visite sur les rivages méditerranéens, car son intérêt va également largement aux collections naturalistes. Dans ses rapports, Brigham porte un regard critique sur la manière française de présenter les collections ethnographiques et sur l'organisation des musées. L'analyse de ses archives apporterait sans doute un éclairage pertinent à l'histoire de ces institutions ${ }^{11}$.

Felix Speiser et Fritz Sarasin, du Museum für Völkerkunde de Bâle (Suisse), s'intéressent respectivement aux collections du Vanuatu et de Nouvelle-Calédonie conservées en France, avant de rédiger leurs ouvrages de synthèse (Speiser, 1923 ; Sarasin, 1929). Sarasin circule notamment dans les muséums de La Rochelle, du Havre, de Toulouse ou de Bordeaux. Plusieurs relevés dessinés des collections du muséum du Havre sont visibles dans les planches illustrées de l'ouvrage de Speiser.

Malgré des évolutions méthodologiques continues, le chemin vers une meilleure connaissance des collections océaniennes est demeuré sinueux. La Seconde Guerre mondiale impose la mise en caisse des œuvres, leur déplacement et, pour certaines, leur disparition - partielle ou complète - sous les bombardements, comme celles du musée des Beaux-Arts de Brest en 1941, du musée de l'Université de Caen et du musée Berthoud de Douai en 1944 (voir Hoffmann, ce volume, pp. 115-126).

\section{Le temps des inventaires}

À partir des années 1950, les initiatives nationales et régionales d'inventaire accompagnant la réorganisation des musées, annoncent la période du réveil des collections. L'ordonnance du 13 juillet 1945 et son décret du 30 août 1945 confient à l'inspection générale de la Direction des musées de France, nouvellement créée, l'inventaire des musées classés et

9. Sur la généalogie des musées nationaux qui ont conservé aux $\mathrm{XIX}^{\mathrm{e}}$ et $\mathrm{XX}^{\mathrm{e}}$ siècles des collections océaniennes, voir le schéma synthétique de Jacquemin $(2000:$ 42) et Leclerc-Caffarel et Servain-Riviale dans ce volume (p. 164).

10. Pour une liste complète des typologies de musées conservant des collections océaniennes, voir Boulay (2007). Plus largement, sur la diversification des typologies de musées, voir Pomian (2021: 47-50).

11. On pourrait y joindre l'étude des archives de Peter H. Buck, Kenneth H. Emory et Margaret Titcomb qui ont étudié méthodiquement divers corpus muséaux français entre la Première Guerre mondiale et 1937 (Laroche, 1945 : 55). 
contrôlés. Du côté des musées sous tutelle du ministère de l'Instruction publique, les premiers recensements des collections ethnographiques furent effectués notamment par E.T. Hamy, dans le contexte de la création du MET (Hamy, 1880). Puis, avec le décret du 27 avril 1948 est créé un service de muséologie au sein du Muséum national d'histoire naturelle, dont les missions s'apparentent à celles de la Direction des musées de France (Pequignot, 2016 : 2-3). Des collaborations entre les deux institutions sont attestées (Faublée, 1954). En 2002, la loi relative aux Musées de France, portée par le ministère de la Culture, définit le cadre réglementaire du récolement décennal, donnant une feuille de route claire aux responsables de musées.

Trois femmes vont mettre en œuvre le recensement systématique des collections océaniennes. La première, Marie-Charlotte Laroche, liste dans son article fondateur de 1945, dix-sept établissements conservant des fonds océaniens. Son attention se porte essentiellement sur les collections originaires de Nouvelle-Calédonie ${ }^{12}$ (1953a, 1964) et notamment sur celles conservées à Toulouse (1953b) et à Rouen (1953c). Elle se rend à Pithiviers pour y examiner le cercueil kanak (Laroche, 1964) ainsi qu’au muséum du Havre (Laroche, 1966).

Puis, ce mouvement initial s'amplifie avec une seconde chercheuse, Anne Lavondès, ethnologue à l'orstom. Afin de préparer les collections du futur musée de Tahiti, dont elle devint ensuite la première directrice, Anne Lavondès se rendit, entre 1974 et 1993, dans près d'une trentaine d'institutions et y établit les inventaires descriptifs des corpus polynésiens et océaniens. De cette façon, elle applique ce qu'elle a appris auprès d'André Leroi-Gourhan au CFRE (Centre de formation à la recherche en ethnologie) du musée de l'Homme. Elle a notamment recours aux fiches muséographiques descriptives, étudiées lors des travaux pratiques dirigés par Hélène Balfet (Lavondès, 2010). Outre sa thèse (1973), certaines de ses études sont publiées, sous forme d'ouvrages, comme l'inventaire du muséum de Grenoble (1990), ou d'articles (pour la collection de Cherbourg par exemple, 1976), ou bien encore remis comme rapport aux musées concernés (Lavondès, 1976, 1978, 1986, 1990, 1994). Sa connaissance approfondie des cultures polynésiennes et sa rigueur méthodologique font de son travail une ressource précieuse pour les études actuelles du patrimoine océanien et polynésien.

Au début des années 1990 émergent les travaux d'une troisième femme, Sylviane Jacquemin, qui a travaillé avec Roger Boulay (1990-1991, 2015). Sylviane Jacquemin soutint une thèse de l'école du Louvre, intitulée «Objets des mers du Sud : Histoire des collections océaniennes dans les musées et établissements parisiens, $\mathrm{XVIII}^{\mathrm{e}}-\mathrm{Xx}{ }^{\mathrm{e}}$ siècles " (1991). En se consacrant à l'histoire complexe des établissements parisiens, elle mit en place les méthodes et l'usage des sources (archives, inventaires, observations des artefacts...) nécessaires à l'étude du parcours des objets (thématique aujourd'hui réactualisée). Ce travail, étendu aux recherches au sein des collections de province (elle a identifié en 1993 les objets de l'expédition d'Entrecasteaux conservés à Dunkerque, $c f$. Jacquemin, 1994) font aujourd'hui référence.

Sylviane Jacquemin et Roger Boulay ont réalisé les inventaires des collections océaniennes des musées du Nord-Pas-de-Calais (Boulay, 1990), bénéficiant du soutien de l'Inspection générale des musées classés et contrôlés et de l'Association des conservateurs de la région Nord-Pas-de-Calais. Cette démarche aboutit à l'exposition «Océanie. La découverte du paradis. Curieux, navigateurs et savants» (1997). L'Association des conservateurs des musées des Hauts-de-France produisit également une base de données, mise en ligne sur son site (https://www. musenor.com/).

\section{Informatisation et mise en réseaux}

De par la complémentarité de leurs travaux, chercheurs, professionnels des musées, enseignants et étudiants commencent à s'établir en réseau, permettant d'aboutir à des résultats fructueux. Ainsi, en 1998, l'exposition "Aquitaine-Océanie, Terres d'échanges " présentée par l'Association des conservateurs des collections publiques de la région Aquitaine au musée d'Aquitaine de Bordeaux, offre dans son catalogue (Merlin-Anglade, 1998) un panorama détaillé des collections océaniennes de Bordeaux, Dax, Libourne et Périgueux. Cette réalisation s'intègre dans un projet original de coopération FranceAllemagne, où se tint ensuite l'exposition sous le nom de "Magie der Südsee ".

Le musée des Beaux-Arts de Chartres initia un travail d'analyse - observation, description, contextualisation - de l'ensemble des artefacts océaniens de sa collection en 1999-2000. Ces études trouvèrent leur concrétisation dans la publication d'un catalogue raisonné des pièces polynésiennes, principalement rassemblées par un seul collectionneur, Louis-Joseph Bouge (Guiot et Stéfani, 2002). Ce travail donna également lieu à un premier recensement des objets wallisiens conservés en métropole, qui permit de réaliser la première exposition en France portant sur l'île Wallis, "Uvea-Wallis, une île pêchée par les dieux " (Guiot, 2000). Au tournant des années 2000, le déploiement d'une documentation informatisée des collections apparait comme l'outil incontournable

12. Elle se rendit par deux fois sur ce territoire, en 1954 et 1956. M.-Ch. Laroche était titulaire d'un certificat d'histoire moderne de l'art, d'ethnologie et d'histoire des religions, ainsi que d'un diplôme de malais de l'École des langues orientales. Attachée au Muséum national d'histoire naturelle de Paris, elle commença sa carrière par une mission avec Marcel Griaule chez les Dogons. Puis elle fut chargée du département d'Océanie du musée de l'Homme de 1940 à 1945. Elle participa de façon active à l'organisation et au fonctionnement de la Société des Océanistes, en tant qu’archiviste, secrétaire générale adjointe puis vice-présidente (Coiffier et Panoff, 1995 : 41-45 ; Langer, 1996 : 319). 
pour la coordination de ce type de travail, la diffusion et la mise en réseau des informations. Sylviane Jacquemin conçoit l'annuaire "Bougainville " (Jacquemin, 1998 : 26) sous la forme d'un dictionnaire des collecteurs et collectionneurs d'art océanien au $\mathrm{XIX}^{\mathrm{e}}$ et dans la première moitié du $\mathrm{XX}^{\mathrm{e}}$ siècle. En 2007, poursuivant le travail de ses prédécesseurs, Roger Boulay, alors chargé de mission à la direction des Musées de France, réalise une importante étude étendue à tous les musées de France pour constituer un annuaire des collections publiques françaises d'objets océaniens (http://www2.culture.gouv.fr/ documentation/joconde/fr/decouvrir/expositions/ oceanie/oceanie_frames.htm) :

" 64017 objets océaniens ont pu être recensés dans 116 établissements français (y compris d'Outremer)»

L'entrée se fait par la ville de conservation (cent y sont inscrites) puis, pour chacune d'entre elle, est fait mention

" des auteurs des collections les plus marquantes, de la date de collecte de ces objets lorsqu'elle est connue. »

S'y trouvent également précisés dates, conditions d'acquisition et origines géographiques des collections. Cet annuaire reste aujourd hui une base de travail pour les étudiants, chercheurs et toute personne intéressée par le patrimoine océanien pour orienter de manière fiable les recherches en musées.

À la même période, l'association MuséoArtPremier crée un site internet (http://www.museoartpremier. com/) comportant une base de données (20052006) donnant accès à la liste de cent cinquante musées français conservant des artefacts extra-européens (collections africaines, américaines, asiatiques et océaniennes). On y trouve toujours « des fichesmusées synthétisant les informations relatives aux collections extra-européennes" (entrée par ordre alphabétique des villes concernées), mais aussi une galerie d'images.

Le musée d'Angoulême met en ligne, à son initiative, la base de données Kimuntu (http://www. annuaire-kimuntu.fr/). Celle-ci concrétise le souhait, formulé lors des « Rencontres du quai Branly 20072008 », par différents responsables de musées conservant des collections extra-européennes

"de rassembler, sur un même espace virtuel, la totalité des structures muséales, universitaires et des personnels qui travaillent, en France, à l'étude des civilisations extraeuropéennes. "

Tel un annuaire, l'entrée dans les rubriques (musées, universités, professionnels) est géographique. Cet ensemble important de ressources est aujourd'hui en cours de réactualisation (voir infra).

Conservateurs et chercheurs poursuivent, dans le cadre de projets d'expositions temporaires notamment, la constitution de bases de données monographiques issues des collections de musées français et étrangers ${ }^{13}$. Soumises à des questions de droits de diffusion, ces bases informatisées restent plutôt confidentielles, mais constituent des ressources importantes. Ces initiatives s'inscrivent dans l'engouement national pour les cultures du monde, impulsé par la création du MQB (1998-2006) devenu MQB-JC le 21 juin 2016.

\section{Quand les musées entrent en chantier}

Les projets de rénovation des musées se développent et permettent d'entreprendre des chantiers de collections, de mettre en place de nouvelles muséographies ainsi que des projets scientifiques et culturels à même de repenser la place des objets océaniens en contexte muséal. Le muséum de La Rochelle entame un processus de réorganisation et de rénovation à partir de 1996, pour ré-ouvrir en 2007, « autrement valorisé » (Patole-Edoumba, ce volume, pp. 21-34). L'Océanie est d'ailleurs l'aire culturelle choisie pour la première exposition temporaire de l'institution, "Tai Kahano, pirogue des îles Marquises ». Le musée Hèbre de Saint-Clément (Rochefort) inaugure une nouvelle muséographie en 2006 présentant notamment un ensemble conséquent d'œuvres aborigènes d'Australie, ainsi qu'un espace consacré à la culture kanak, intégrant tradition et renouveau (Stéfani, ce volume, pp. 61-76). De même, le muséum d'histoire naturelle du Havre s'engage dans une nouvelle dynamique qui aboutit au reconditionnement des collections de dessins de Charles-Alexandre Lesueur liées à l'expédition scientifique dirigée par N. Baudin en 1800 en Australie. Un cabinet d'art graphique, dédié à la mise en valeur de ce fonds fragile grâce à des rotations d'expositions, est aménagé en 20062007. En 2007 toujours, c'est au tour du musée de Boulogne-sur-Mer de dévoiler ses nouvelles vitrines extra-européennes. Le muséum d'histoire naturelle de Toulouse ferme en 1997. Le déménagement des collections du bâtiment ancien, lors du projet de rénovation, permit de renouveler l'intérêt pour le fonds extra-européen. Il est réouvert en 2008 et des spécialistes s'y succèdent, depuis, pour des études spécifiques du corpus océanien (voir Bonvin-Pochstein et al., ce volume, pp. 49-60). Cette même année, le musée d'Angoulême inaugure, après trois ans de rénovation, un nouveau parcours de visite, intégrant pour l'Océanie une vitrine consacrée aux curios et à l'imagerie européenne.

\section{Un patrimoine océanien dispersé}

Parallèlement, un travail de grande ampleur prend naissance en Océanie. Dès les années 1980, JeanMarie Tjibaou émet l'idée d'un recensement du patrimoine matériel kanak dispersé dans les musées du monde et, en particulier, dans les musées français. Roger Boulay, en lien avec l'Agence de développe-

13. En préparation de l'exposition «Sepik. Arts de Papouasie-Nouvelle-Guinée » (Berlin, Paris, 2015), Philippe Peltier, Christian Kaufmann et Marcus Schindlbeck mirent au point une base de données à accès restreint consacrée aux arts du fleuve Sepik. 
ment de la culture kanak et le centre culturel Tjibaou à Nouméa, produit une base de données des principaux musées en France et en Europe qui conservent le patrimoine kanak. Ce projet a abouti notamment à l'exposition «De Jade et de Nacre, Patrimoine Kanak ", présentée à Nouméa en 1990 et à Paris en 1991. Le souhait de J.-M. Tjibaou prend une forme décisive entre 2011 et 2015, avec l'inventaire du patrimoine kanak dispersé (IPKD) confié à Emmanuel Kasarhérou par le gouvernement de la Nouvelle-Calédonie. Emmanuel Kasarhérou est chargé d'établir un programme scientifique et technique d'inventaire des objets du patrimoine kanak et met en place une équipe de quatre personnes pour le mener à bien. Soixante-trois musées visités, 16600 objets examinés, 919 fiches biographiques de collecteurs/donateurs... au regard de ce qui était connu auparavant, l'avancée est remarquable.

« Le bilan scientifique del'IPKD est d'abord l'augmentation considérable du corpus [...] ; les collections sont composées pour plus d'un tiers d'armes $[. .$.$] ; l'information sur les$ provenances relève de l'exceptionnel et non de la norme. " (Kasarhérou, $2021: 13$ )

Cet admirable enrichissement des connaissances introduit également de nouveaux paradigmes pour penser les objets, comme les collections : ainsi en est-il de l'articulation entre "patrimoine dispersé " et " patrimoine partagé " et de la notion "d'objets ambassadeurs ", introduite par Emmanuel Kasarhérou au début des années 2000 , au sujet d'une tête de monnaie de son propre clan, alors conservée dans les réserves du musée de l'Homme (Boulay, 2021 : 18). L'exposition "Kanak. L'art est une parole ", présentée au MQB en 2013 (Boulay et Kasarhérou, 2013) permit de mesurer l'étendue des savoirs acquis.

Aboutissement de trente-cinq années d'efforts, cet inventaire raisonné d'un patrimoine insulaire fait figure d'initiative pionnière inspirant d'autres programmations. En 2016, le gouvernement de la Polynésie française met en place son projet d'inventaire des collections polynésiennes dans les musées nationaux et étrangers. Initié par Théano Jaillet, alors directrice du musée de Tahiti et des îles Te Fare Manaha (MTI), avec le soutien du ministère de la Culture de Polynésie française et de son ministre Heremoana Maamaatuaiahutapu, Véronique Mu-Liepmann, conservatrice du MTI de 1982 à 2011, est missionnée pour réaliser des fiches d'objets destinées à alimenter une base de données (créée en 2018) des objets polynésiens dispersés au sein de diverses collections. Miriama Bono, actuelle directrice du MTI, poursuit l'initiative, fixée comme prioritaire par le ministère de tutelle, et en 2019, Hélène Guiot, spécialiste de la culture matérielle polynésienne, rejoint le projet. Les contacts entre le MTI et les musées visités (quarantetrois à ce jour) se structurent afin de construire un réseau pour la mise en œuvre de futures collaborations. Les objectifs de ce programme visant à identifier et à localiser le patrimoine de Polynésie française dispersé s'inscrivent dans une perspective d'études et de colla- boration scientifiques avec les différentes institutions sollicitées. La plateforme numérique mise en place deviendra une ressource utile à la fois aux chercheurs, aux acteurs du patrimoine et à tous les Océaniens qui s’y intéresseront.

\section{Un travail sur le temps long, une accélération récente : multivocalités}

Une réorientation des missions et des attentes quant à la responsabilité sociale et politique des musées se cristallise en 2019, lors de l'assemblée générale extraordinaire de l'icom (International council of Museums), réunie à Tokyo le 7 septembre pour le vote d'une nouvelle définition des musées :

\begin{abstract}
«les musées sont des lieux de démocratisation inclusifs et polyphoniques, dédiés au dialogue critique sur les passés et les futurs. Reconnaissant et abordant les conflits et les défis du présent, ils sont les dépositaires d'artefacts et de spécimens pour la société. Ils sauvegardent des mémoires diverses pour les générations futures et garantissent l'égalité des droits et l'égalité d'accès au patrimoine pour tous les peuples. Les musées n'ont pas de but lucratif. Ils sont participatifs et transparents, et travaillent en collaboration active avec et pour diverses communautés afin de collecter, préserver, étudier, interpréter, exposer, et améliorer les compréhensions du monde, dans le but de contribuer à la dignité humaine et à la justice sociale, à l'égalité mondiale et au bien-être planétaire. »
\end{abstract}

Si cette définition affermit un cap déjà pris depuis les années 2000 par les musées conservant des collections extra-européennes, remettre au centre des pratiques la démarche collaborative, la multi-vocalité des discours et des dialogues critiques autour des objets ne va pas encore de soi dans les musées français. La définition ICOM de 2019 n'est d'ailleurs pas encore votée à ce jour, dans l'attente d'une reformulation. Cet appel au changement se double d'une urgence nouvelle pour les équipes muséales, fruit des constats sur l'impérieuse nécessité d'engager au sein des musées "une nouvelle éthique relationnelle " (Sarr et Savoy, 2018). Dans le cas des objets océaniens, la recherche de provenance et le travail biographique n'ont pas, on l'a vu plus haut, attendu ces dernières injonctions politiques. Rappelons qu'en ce début de $\mathrm{xxI}^{\mathrm{e}}$ siècle, les restitutions d'objets ont activement concerné les collections océaniennes, avec notamment le retour des têtes momifiées māori, mokomokai (Gagné, 2012 ; Boulay, 2012 ; Peltier et Mélandri, 2012).

Néanmoins, le regard distancié et critique sur les conditions des collectes françaises dans le Pacifique à partir du XviII e siècle n'est que peu lisible aujourd'hui dans les études, comme le soulèvent $S$. Bonvin, M. Dufau et L. Granier dans ce volume, à propos de l'expédition de La Flore et de "l'enlèvement " d'un moai à l'île de Pâques ${ }^{14}$. De même, l'exigence éthique légitime de travailler à la compréhension des collections océaniennes de manière collaborative et inclusive est encore timide au sein des études muséales 


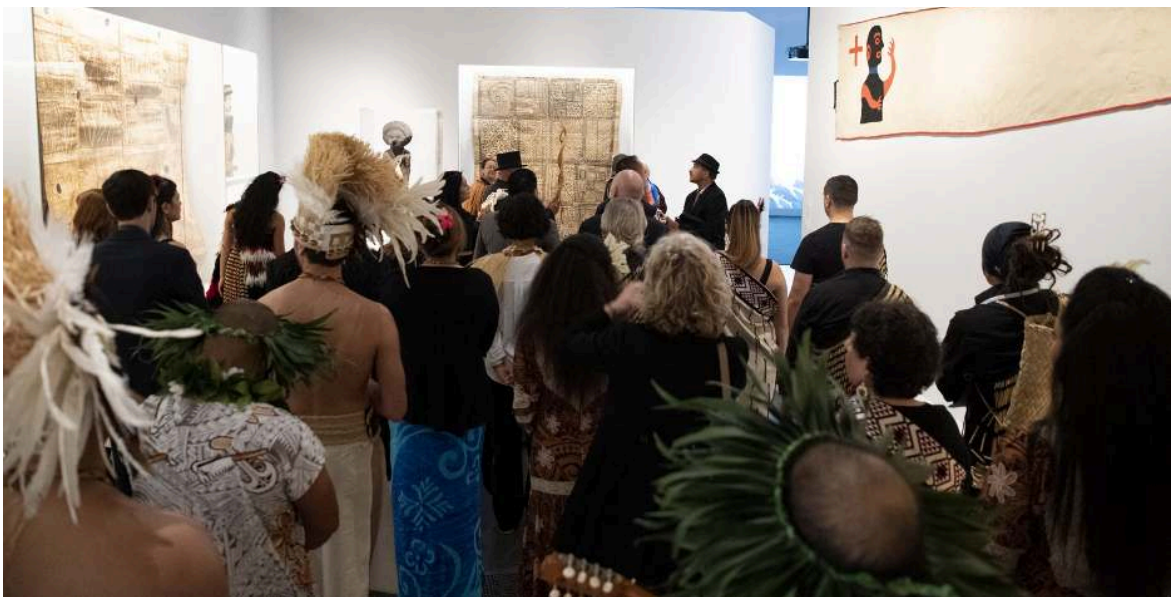

Рното 3. - Cérémonie d'ouverture blessing ceremony dans le cadre du vernissage de l'exposition Océanie - 12 mars-7 juillet 2019 (cliché Thibaut Chapotot, (C) MQB-JC)

françaises. Ces collaborations se développent de plus en plus dans les musées en région (Rochefort, La Rochelle, Toulouse, Bordeaux pour quelques exemples), mais elles sont notamment confrontées aux moyens que ce dialogue implique et qui ne sont pas toujours dans les priorités budgétaires des collectivités qui administrent les musées. L'idée d'un patrimoine partagé fait son chemin, compris en tant qu'un don des musées appelant un contre-don en matière de savoirs autochtones. Les musées comme les communautés récoltent mutuellement les bénéfices de leur collaboration: informations techniques vernaculaires et symboliques sur les objets et leurs usages pour les musées; (re)découverte, réappropriation, (re) construction identitaire et renouveau culturel pour les communautés.

Par comparaison, il est intéressant d'observer Outre-Manche le rôle moteur du musée d'Archéologie et d'Anthropologie de l'université de Cambridge et celui du British Museum en matière de recherche historique et muséographique collaborative. Entre 1973 et 1986 déjà, sous l'égide de Peter Gathercole alors au musée de Cambridge, avait été dressé un inventaire sommaire des collections océaniennes conservées en Australie, en Nouvelle-Zélande, au Royaume-Uni, aux États-Unis et au Canada, dans le cadre du Oceanic Cultures Project (Specht et Bolton, 2005), projet UNESCO motivé par le mouvement des indépendances dans le Pacifique et la critique des leaders politiques sur le manque d'accès à leur patrimoine dispersé. Peter Gathercole soulignait :

"artefact documentation became politically necessary as well as academically proper. " (ibid. : 58)

Plus récemment, trois projets de recherche, menés dans un temps resserré, se sont distingués par leur envergure: Artefacts of encounter (2010-2013; Thomas et al., 2016), Fijian Art Research Project (2011-2013; http://www.fijianart.sru.uea.ac.uk/), Pacific Presences: Oceanic Art and European Museums" (2014-2018; https://cordis.europa.eu/project/rcn/107885/ factsheet/en). Tous trois s'articulaient autour de l'analyse critique des contextes de constitution des collections d'art océanien en Europe entre le $\mathrm{xVIII}^{\mathrm{e}}$ et le $\mathrm{xXI}^{\mathrm{e}}$ siècle, de l'étude historique et matérielle de corpus d'objets et, pour certains, d'une attention portée aux nouvelles approches muséographiques de ces collections. D'un point de vue méthodologique, outre les chercheurs spécialisés recrutés pour explorer les archives et visiter les réserves des grands musées à travers le Royaume-Uni et l'Europe (Carreau et al., 2018), ces recherches impliquaient la présence de spécialistes ou de détenteurs de savoirs originaires du Pacifique ainsi que d'artistes contemporains qui se consacraient à une restitution créative des processus de recherche.

\section{Présentation des contributions}

Loin de proposer une synthèse, ce dossier constitue, par la confrontation de cas d'études, un point d'étape au sein d'une histoire conjointe des musées et de la recherche. Les onze textes présentés dans ce dossier, réunis selon quatre ensembles thématiques, reflètent l'actuelle diversité des problématiques et des approches autour des collections océaniennes. Ils sont emblématiques des protagonistes à l'œuvre dans ce travail au long cours de collecte, de conservation, d'étude et de valorisation des objets. La pluridisciplinarité et la complémentarité des compétences nourrissent des travaux qui prennent souvent l'aspect de

Рното 4. - Week-end et soirée Before organisés par le MQB-JC pendant l'exposition Océanie, 28-30 juin 2019 (cliché Valérie Jacob, (C) MQB-Jc)

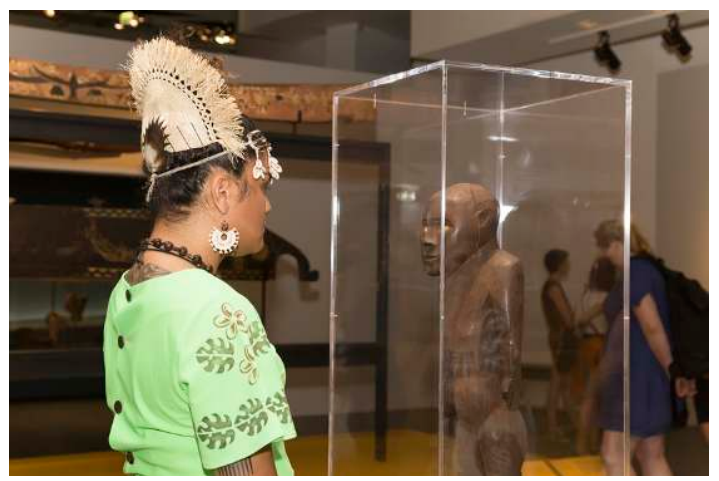

14. Cette tête de moai est aujourd'hui présentée dans le hall d'accueil du MQB-JC (inv. 71.1930.35.1). 
minutieuses enquêtes de traçabilité matérielle et de biographies, fréquemment éparses ou incomplètes à l'origine.

\section{Enjeux contemporains des musées}

Le premier ensemble d'articles donne la parole à cinq professionnelles de musées. Retraçant la constitution historique des collections dont elles ont la responsabilité, elles témoignent du parcours mouvementé des objets jusqu'aux musées qui les conservent aujourd'hui. Leurs textes mettent en perspective les questions de méthodologie de recherche, de politique d'acquisitions, de muséographie et de médiation auprès des publics.

Elise Patole-Edoumba présente le processus de patrimonialisation des collections polynésiennes du muséum de La Rochelle en s'appuyant sur les réseaux d'intentions qui entourent un objet. Ce processus se fait l'écho toujours vivace des principes d'agentivité (agency) développpés par Alfred Gell (1998 : 12-51), en particulier de son équation à combinaisons multiples entre producteur, utilisateur(s) et récipiendaire(s) d'une œuvre. La personnalité du conservateur Étienne Loppé (1883-1954) et ses interconnexions avec un milieu érudit et bourgeois - à la fois local et national - est intéressant à ce titre. Homme-orchestre de l'enrichissement des collections du muséum (Elise Patole-Edoumba, fig. 1, p. 26), il définit l'objet comme une preuve matérielle de l'action coloniale de la France, un témoin scientifique et technologique d'une culture, une création non dénuée d'une certaine esthétique, et décline une muséographie-miroir de ces niveaux de sens. S'agissant des acquisitions actuelles du musée, la tension entre un souci d'enrichir les témoins passés d'une histoire de l'ici et de l'ailleurs, et la volonté d'ouvrir la voie aux enjeux actuels de globalisation au travers d'œuvres contemporaines est manifeste.

Théano Jaillet, quant à elle, développe une réflexion interrogeant l'identité même des institutions, notamment lorsqu'elles changent d'appellation, comme le musée de la Castre de Cannes, devenu récemment musée des Explorations du Monde (MEM) ${ }^{15}$. Son article retrace une histoire des acquisitions, faite de rencontres et d'opportunités - c'est souvent le cas -, et de quêtes de l'objet manquant pour atteindre une forme de complétude des collections à mêmes de représenter les sociétés du Pacifique. Le choix actuel du musée vise à renforcer la contextualisation historique de la collecte d'Edmond de Ginoux de la Coche, à l'origine du fonds océanien du musée, suite à l'achat d'un ensemble de dessins d'Adèle de Dombasle, compagne de voyage de Ginoux. Cette démarche est fructueuse pour le développement de coopération de recherche entre institutions - une étude comparée des dessins d'Adèle de Dombasle conservés au MQB-JC ayant été menée dans le cadre de cette acquisition afin de documenter ces fragments d'une même collection. On notera également pour Cannes, comme pour La Rochelle, l'acquisition continue de tapa, ces étoffes océaniennes confectionnées à partir de liber battu. Conservés en nombre et bien documentés (Kooijmann, 1972; Neich et Pendergast, 1998 ; Guillaut et al., 2009 ; Lennard et Mills, 2021), les tapa constituent un axe d'enrichissement des collections souvent retenu pour dépasser la dichotomie entre tradition et modernité et dresser dans les vitrines une brève histoire des techniques et des styles.

Puis Sylviane Bonvin-Pochstein, Magali Dufau et Lành Granier parcourent l'histoire de la muséographie du muséum d'histoire naturelle de Toulouse depuis les années 1950, dressant le constat d'une difficile adéquation entre une muséographie conceptuelle - structurée autour du thème des grandes fonctions du vivant -, un accrochage esthétisant des œuvres, et ce qu'en comprend - ou pas - le public. À travers un regard critique sur les discours et les pratiques muséographiques, puis une présentation des démarches collaboratives développées à Toulouse, elles interrogent la façon dont le musée peut opérer la décolonisation de son discours. La présentation des institutions dont il est question dans cette partie montre que l'amorce d'une démarche inclusive, autour d'un patrimoine partagé, est désormais engagée.

\section{Du terrain au musée}

Suivant la chronologie des explorations du $\mathrm{XIX}^{e}$ siècle puis des missions scientifiques au tournant $\mathrm{du} \mathrm{Xx}^{\mathrm{e}}$ siècle, les trois textes de ce second ensemble présentent des collectes de terrain et le riche matériau documentaire qui leur est associé. Cette richesse a motivé le parti-pris muséographique du musée Hèbre à Rochefort, porté par Claude Stefani et centré sur l'histoire de la collecte historique. L'étude approfondie des sources primaires laissées par René-Primevère et Pierre-Adolphe Lesson démontre l'intérêt d'une exploitation pièce à pièce, ligne à ligne de ces archives pour la contextualisation des objets. Cette recherche minutieuse déconstruit en partie l'idée que les collectes liées aux voyages d'exploration n'ont que peu documenté la provenance et la valeur des objets. Les deux articles suivants de Margot Duband et Marie Adamski sont liés à la naissance des musées d'ethnographie au tournant des $\mathrm{XIX}^{\mathrm{e}}-\mathrm{XX}^{\mathrm{e}}$ siècles. Ils ont en commun d'aborder deux missions : celles de M. Archambault (1909-1910) et de P. ReyLescure (1929-1933), dont les objets rapportés sont aujourd'hui réunis au MQB-JC et sont caractérisés par des typologies matérielles et documentaires diverses,

15. On citera en complément l'intitulé du projet scientifique et culturel (PSC) 2020-2025 du musée d'Aquitaine, "un muséemonde, de Bordeaux et d'Aquitaine " ou encore le vote, ouvert en 2021 par le musée d'Ethnographie de Genève, pour un changement du nom du musée, mieux à même d'incarner les nouvelles orientations du plan stratégique 2020-2024, centré sur la décolonisation de cette institution. 
mais peu étudiées jusqu'ici. Les trois textes donnent le détail de la chaîne opératoire de la collecte et de ses moyens logistiques. Tandis que les témoignages des frères Lesson soulignent les modes d'échanges asymétriques à l'œuvre dans l'acquisition des artefacts océaniens, les archives Archambault et Rey-Lescure nous apprennent à quel point le MET n'a que peu soutenu financièrement ces missions, faute de moyens adaptés ou considérant certaines des collectes d'enrichissement du nouveau musée comme des partenariats étendus à un vaste réseau de bénévoles sur le terrain (voir aussi Laurière, 2017 : 409; Laurière et Peltier-Caroff, 2017 : 915-919). Ainsi, la mission de M. Archambault est directement financée par le ministère de l'Instruction publique, tandis que l'envoi des caisses d'objets collectés par P. Rey-Lescure n'est possible que grâce au soutien de l'administration coloniale française in situ. M. Adamski précise le rôle des missionnaires religieux dans la connaissance des sociétés océaniennes et de leur culture matérielle. Au détour de ces missions transparait l'émulation entre musées européens à cette période, le musée d'ethnographie de Bâle étant alors une institution particulièrement active (F. Sarasin pour la Nouvelle-Calédonie et F. Speiser pour le Vanuatu). Une étude croisée de ces collectes européennes, quasi contemporaines, serait intéressante à mener pour identifier les positionnements scientifiques à l'œuvre.

\section{L'effervescence de la recherche}

Le troisième ensemble de textes témoigne du dynamisme des recherches sur les collections océaniennes au sein des formations universitaires depuis une vingtaine d'années. Ces travaux récents mettent l'accent sur des collections méconnues ou mal identifiées, constituent de premiers corpus documentaires, recensent, organisent et publient des sources archivistiques encore non traitées. Depuis les années 2000 , près de trente travaux de recherche de l'école du Louvre ont ainsi été consacrés à l'étude des collections océaniennes conservées en France et à leur valorisation (voir bibliographie). Afin d'appréhender dans leurs spécificités les collections muséales extraeuropéennes, les étudiants empruntent des parcours hybrides, associant en parallèle ethnologie ${ }^{16}$, histoire des arts océaniens et muséologie ${ }^{17}$. Ces formations reflètent les profils qui ont incarné, depuis le début $\mathrm{du} \mathrm{Xx}^{\mathrm{e}}$ siècle, la recherche autour des collections océaniennes en France : celui du chercheur - ethno- logue, archéologue, historien, linguiste - rattaché à un laboratoire ou une université, et celui du professionnel de musée - conservateur ou responsable de collection -, les deux se conjuguant parfois.

Dans les articles regroupés ici, l'enquête permettant de remonter le fil chronologique d'une collection, existante ou disparue - car il faut aussi documenter l'absence -, est faite de tâtonnements, de pistes à lancer, qui révèlent parfois des trouvailles ou restent à l'état d'hypothèses, voire d'impasses, attendant la manifestation inattendue de descendants non identifiés jusqu'ici, la venue de spécialistes ou que d'autres collections alentours amènent de nouvelles orientations d'investigation. Ces cas d'étude puisent dans le tissu local pour raconter le goût des amateurs érudits, $S$. Berthoud et E. Jomard à Douai (Marie Hoffman), des sociétés savantes telle la Société d'émulation du Bourbonnais à Moulins (Margaux Chataignier) et des collecteurs "en herbe " comme les époux Le Rat à Alençon (Soizic Le Cornec), autant de catégories d'acteurs pourvoyeuses en objets de nombreux musées régionaux. Passé ce premier constat, se dessinent des parcours plus atypiques, telle une femme collectrice, en Mélanésie, à l'aube du Xx $x^{e}$ siècle - le fait est assez rare pour être noté (S. Le Cornec). Les méthodologies pionnières dans le classement des collections du musée Berthoud conduisent à reconsidérer, quant à elles, le statut de "musée-laboratoire" souvent réservé aux seuls établissements nationaux. On voit aussi à quel point ces collections, longtemps invisibilisées, sont révélées et valorisées auprès des publics, sous l'impulsion des démarches d'inventaires des patrimoines dispersés tels l'inventaire du patrimoine kanak dispersé ${ }^{18}$ et l'inventaire des collections de Polynésie française.

\section{Au coeur de l'objet}

Les objets océaniens conservés et exposés dans les musées incarnent, par leur matérialité intrinsèque, des trajectoires et des valeurs diverses qui les ont liés et soustraits tout à la fois à leur(s) culture(s) d'origine. L'étude visuelle des objets constitue en ce sens une étape méthodologique incontournable dans l'étude des collections. Dans cette approche, l'irruption des sciences dures a permis d'améliorer notre compréhension des objets tout en garantissant leur préservation et leur transmission, les instruments d'analyse utilisés étant de moins en moins invasifs. Dans le dernier ensemble d'articles, on découvrira

16. Universités de Paris I, de Paris Nanterre, d'Aix-Marseille, de Strasbourg, de Bordeaux, de Polynésie française et de NouvelleCalédonie.

17. Aux côtés du pionnier cycle 2 en muséologie de l'école du Louvre suivi de la formation initiale des conservateurs au travers de l'Institut national du Patrimoine créé en 1990, ont éclos: les master Patrimoine et musées des universités Bordeaux Montaigne, Lyon-III, Paris I, Paris 8 et Paris Nanterre, Paul Valéry de Montpellier, universités de Brest, de Pau et des Pays de l'Adour, de Reims ; le master Muséologie des sciences de la nature et de l'homme au Muséum national d'histoire naturelle ; le master Ethnologie-parcours muséologie, patrimoine immatériels et collections à l'université de Strasbourg ; le master Histoire, Civilisations, Patrimoine, Parcours muséologie de l'Université Haute-Alsace ; le master Muséologie-muséo-expographie de l'université d'Artois.

18. L'exposition «Trajectoires Kanak. Histoires de voyages en Nouvelle-Calédonie » présentée au musée Anne de Beaujeu en 2017 2018, trouve écho dans celle du musée du Berry, à Bourges, "Enquête sur une collection Kanak " (1/7/2020-11/1/2021). Toutes deux ont bénéficié de l'expertise de Roger Boulay, puis d'Emmanuel Kasarhérou pour l'analyse matérielle et historique des collections exposées. 
une nouvelle fois (voir M. Hoffmann), au travers de l'article de F. Servain-Riviale et S. Leclerc-Caffarel, que c'est par son absence qu'un objet peut motiver une enquête de fond. Dans un travail à rebours, il aura fallu partir des indices visuels de représentation de l'objet manquant, des archives des voyages et des inventaires croisés du premier musée de la Marine du Louvre pour remonter la piste du plus ancien spécimen de contenant en algue tasmanien encore conservé.

Le déploiement de techniques d'analyse avancées achève de confirmer l'hypothèse. Cet apport considérable des sciences est le fruit des programmes développés depuis une dizaine d'années par les chargés d'analyses scientifiques du MQB-JC, en lien avec les responsables des collections ${ }^{19}$. Si cet appareillage est encore peu accessible pour les collections des musées en région, certains ont pu en bénéficier dans le cadre de partenariats d'exposition (voir Pochstein et al.).

Dans ces études, l'œil est à l'œuvre, mais aussi la main. Ou plutôt plusieurs mains, qu'il s'agisse de la fabrication des manteaux māori à bordures kaitaka étudiés par L. Renard, comme des récipients à eau tasmaniens (Servain-Riviale et Leclerc-Caffarel). L'étude de ces deux types d'artefacts souligne l'importance des savoir-faire féminins dans la production d'objets exceptionnels, ainsi que leur rôle de premier plan dans la revitalisation culturelle contemporaine. Pour une meilleure compréhension du geste technique et des enjeux symboliques et sociaux qui imprègnent ces biens de prestige, L. Renard s'est tournée vers l'apprentissage pratique, une façon d'engager la collaboration avec les expertes-tisseuses māori qui l'accompagnent en retour dans le développement de sa recherche. Ces derniers articles font la démonstration qu'une éthique de la recherche sur les collections muséales extra-européennes est primordiale. L'étude historique approfondie, dès lors qu'elle est assortie d'une démarche collaborative auprès des détenteurs des savoirs et des savoir-faire, ou des artistes à même de réinterpréter les modèles traditionnels, démultiplie les niveaux de significations des objets. Ces collaborations et les productions intellectuelles et matérielles qui en résultent font également surgir de l'émotion, une valeur au cœur de l'efficacité esthétique des objets du Pacifique qui favorise leur partage avec le public des musées (Jeudy-Ballini, 1999).

Que dirait Marie-Charlotte Laroche, soixantequinze ans après ses premiers constats et ses grands espoirs? Le chemin parcouru est manifeste, l'avancée des connaissances indiscutable. La démarche suit le temps long des institutions, dont les mutations, ajoutées à la structuration de la réglementation en matière de gestion des collections, ont permis ces vingt dernières années une accélération des recherches. La convergence des initiatives est en route, bien qu'encore trop éclatée pour envisager une organisation à grande échelle de la recherche. Le service des musées de France/direction générale des Patrimoines a néanmoins impulsé depuis 2019 divers projets dédiés à l'histoire des collections extra-européennes, en partenariat avec l'Institut national de l'histoire de l'art (INHA). Une journée d'étude intitulée "Inventaire, documentation, recherche de provenance: quels enjeux pour les collections extra-occidentales des musées de France ? " fut organisée le 7/10/2019 avec le MQB-JC, qui présenta à l'occasion son plan d'action en matière de recherches sur l'origine et l'histoire des collections qu'il conserve (https://www.culture.gouv. $\mathrm{fr} /$ Sites-thematiques/Musees/Actualites/Publicationde-la-journee-d-etude-sur-les-collections-extra-occidentales-des-musees-de-France-Paris-07-10-2019). Réminiscence des rencontres professionnelles organisées par ce même musée national dans les premières années de son ouverture, ce partenariat confirme le rôle de " grand département " assigné au MQB-JC ${ }^{20}$.

Plus récemment, le séminaire Parcours d'objet. Études de provenance des collections d'art "extraoccidental " inauguré en 2020 à l'InHA et porté par V. Lefebvre, conservateur général au musée Guimet et E. Salaberry, directrice des musées municipaux d'Angoulême, vise à faire état des travaux de recherche sur ces collections, à donner des outils méthodologiques, à valoriser des enjeux, des méthodes et des ressources ou de formuler des besoins (https://www.inha.fr/ $\mathrm{fr} /$ recherche/programmation-scientifique/en-20202021/parcours-d-objets-etudes-de-provenance-descollections-d-art-extra-occidental.html). Développé à l'attention des conservateurs et des chercheurs, ce séminaire a consacré, le 7/1/2021, l'une de ses séances au patrimoine océanien (https://www.youtube.com/watch? $v=$ nrxG93K2wp4\&list=PLsl8NW zVv6T0ySesI2A5fvyvG5MxynGBB\&index=2\&t=5 028s). L'inHa porte en parallèle le projet « Monde en musées, cartographie des objets africains et océaniens dans les collections publiques françaises " (https:// inhadoc.hypotheses.org/1791). Si ce projet constitue une forme de sauvegarde de la base de données Kimuntu (supra p. 11), il la transforme en un nouvel outil de cartographie des collections et permet de valoriser une des collections d'art africain dans le cadre du projet "Vestiges, indices, paradigmes : lieux et temps des objets d'Afrique (XIV ${ }^{e}-\mathrm{XIX}^{\mathrm{e}}$ siècles) ", également développé à l'inHa. L'école du Louvre, forte du nombre important d'étudiants engagés dans l'étude des collections océaniennes en master et doctorat, cherche également à nourrir les recherches en soutenant l'attribution d'une bourse post-doctorale dédiée

19. Dans le domaine océanien, plusieurs analyses par imagerie 3D ont déjà été mises en œuvre : étude de la structure interne des masques de deuilleur kanak de Nouvelle-Calédonie (2012), des techniques de fabrication et des matériaux constitutifs des éventails tahi'i des îles Marquises (Kerfant, Mélandri et Moulherat, 2019) ainsi que pour l'identification du présumé fragment de ceinture de chefferie tahitienne, maroura (Alevêque, 2018).

20. En tant que grand département (voir l'article R 422-1 du code du patrimoine), le MQB-JC est l'institution nationale ressource pour les musées du territoire concernés par des collections extra-occidentales. À ce titre, les responsables de collection sont amenés à renseigner des identifications d'objets, soutenir des projets d'acquisition, contribuer à des projets de recherche. 
à l'histoire des collections océaniennes et de leur muséographie (projet en cours de développement). Cet axe d'étude serait à approfondir en rassemblant les corpus iconographiques témoignant des muséographies successives conservés dans les musées, dressant les chronologies des expositions, observant les objets qui portent parfois les premiers indices de leur traitement muséographique (de Poulpiquet, 2012), par exemple. La tâche est ardue tant ces ressources sont disparates. Cet axe d'étude contribuerait néanmoins à éclairer les régimes de valeur accordés à ces objets au cours du temps: trophées de collecte, objets-témoin, objets-mémoire, ouvres d'art etc.

Ces projets gagneraient à se fédérer autour d'un porteur global, soutenus par un financement à la hauteur des enjeux et par un développement pluriannuel des recherches. Cela garantirait la réussite d'une telle entreprise, dont on sait qu'elle pourrait d'ailleurs s'étendre à l'ensemble des collections d'Océanie, d'Afrique, des Amériques et d'Asie. Surtout, un tel projet ne peut s'envisager aujourd'hui sans que ne soient invités dans les réserves de nos musées des représentants culturels du Pacifique ou que ne soient conçus des outils de partage à même de diffuser, dans le respect juridique et éthique des prescriptions coutumières propres aux cultures dont nous préservons l'héritage, les ressources et les résultats de ces recherches. Dans une perspective inversée, s'attacher à l'histoire, aux trajectoires et aux enjeux des musées du Pacifique et de ceux qui les ont soutenus et développés reste encore à initier, afin d'offrir un panorama global et renouvelé de cette relation entre objets, collections et musées.

\section{Remerciements}

Nous souhaitons dès l'ouverture de ce dossier remercier vivement les membres du comité de rédaction pour leur implication et leur expertise face à la profusion d'études qui ont été soumises.

\section{BIBLIOGRAPHIE}

(La version en ligne de cet article sera augmentée d'une liste des mémoires de master de l'école du Louvre consacrés aux collections océaniennes, à leur étude et à leur valorisation de 2003 à 2020.)

AlevêQue Guillaume, 2018. Remnants of the "Wallis maro 'ura' (Tahitian feathered girdle): history and historiography, Journal of Pacific history 53, 1, pp. 1-62.

Apollinaire Guillaume, 1991 [1909]. EFuvres en prose complètes, Paris, Gallimard, t. 2, pp. 122-124.

BAERT Annie, 1999. Le paradis terrestre, un mythe espagnol en Océanie. Les voyages de Mendana et de Quiros 1567-1606, Paris, L'Harmattan.
BLAIs Hélène, 2000. Les voyages français dans le Pacifique: pratique de l'espace, savoirs géographiques et expansion coloniale: (1815-1845), thèse de doctorat, Histoire, Paris, EHEss.

Bonnemaison Joël, 1986. La dernière île, Paris, Arléa, оRSTOM.

Boulay Roger, 1990. Actualités d'un débat qui s'éternise : que faire des collections d'objets exotiques, La Lettre de l'OCIM 9, pp. 18-22.

—, 1990-1991. Les collections océaniennes du musée de Boulogne-sur-Mer, Journal de la Sociéte des Océanistes 90, pp. 29-34 (https://doi. org/10.3406/jso.1990.2864).

—, 1997. Le chevalier Leroy de Barde et les cabinets de curiosités anglais, in Océanie. La découverte du paradis. Curieux, navigateurs et savants, Paris, Somogy-Editions d'Art, pp. 33-35.

—, 2007. Annuaire des collections publiques françaises d'objets océaniens (http://www2.culture. gouv.fr/documentation/joconde/fr/decouvrir/ expositions/oceanie/oceanie_frame.htm).

-, 2012. L'initiative du Muséum d'histoire natrelle de Rouen : le contexte local, Journal de la Société des Océanistes 134, pp. 25-27 (https://doi. org/10.4000/jso.6637).

-, 2015. Les collections extra-européennes : France après, La Lettre de l'OCIM 158, pp. 31-34.

—, 2021. Aventure d'inventaire, in Carnets kanak. Voyage en inventaire de Roger Boulay, Paris, musée du quai Branly-Jacques Chirac, pp. 15-23.

Boulay Roger et Emmanuel Kasarhérou (éds), 2013. Kanak. L'art est une parole, Paris-Arles, musée du quai Branly-Actes Sud.

BRIGHAM William T., 1898. Report Report of a journey around the world undertaken to examine various ethnological collections, Occasional papers of the B. P. Bishop Museum 1, 1, pp. 1-72.

- 1913. Report of a journey around the world to study matters relating to museums 1912, Honolulu, Occasional papers of the B. P. Bishop Museum 5,5 .

Carreau Lucie et al., 2018. Pacific Presences: Oceanic Art and European Museums, Leiden, Sidestone press, 2 vol.

Coifrier Christian (éd.), 2001. Le voyage de la Korrigane dans les Mers du Sud, Paris, Hazan.

CoIfrier Christian et Michel Panoff, 1995. Quelques aspects de l'histoire de la Société des Océanistes: un entretien avec M.-C. Laroche, Journal de la Société des Océanistes 100-101, pp. 4155 (https://doi.org/10.3406/jso.1995.1946)

Collectif, 1997. Océanie. La découverte du paradis. Curieux, navigateurs et savants, Association des 
conservateurs des musées du Nord-Pas-de-Calais, Somogy Éditions d'Art.

Delpuech André, 2017. Collectes, collecteurs, collections dans les années trente. Entre théorie ethnologique et réalités pratiques, in André Delpuech, Christine Laurière et Carine PeltierCaroff (éds), Les années folles de l'ethnographie. Trocadéro 28-37, Paris, Muséum d'histoire naturelle, pp. 449-479.

Dias Nélia, 1991. Le musée d'Ethnographie du Trocadéro (1878-1908). Anthropologie et muséologie en France, Paris, éditions du CNRS.

DuPERREY Louis Isidore, 1825-1830. Voyage autour du monde exécuté par ordre du Roi sur la corvette de Sa Majesté, La Coquille, pendant les années 1822, 1823, 1824 et 1825 , Paris.

FAublée Jacques, 1954. Sculptures mochica des îles Macabi, Journal de la Société des Américanistes 43, pp. 149-150 (https://doi.org/10.3406/ jsa.1954.2420).

Gell Alfred, 1998. Art and Agency. An anthropological theory, Oxford, Clarendon Press.

Girard Françoise, 1945. Récentes acquisitions du musée de l'Homme, Journal de la Société des Océanistes 1, pp. 125-128 (https://doi.org/10.3406/ jso.1945.1500).

- 1953, Les riches collections néo-calédoniennes du musée de l'Homme, Journal de la Société des Océanistes 9, pp. 302-306 (https://doi. org/10.3406/jso.1953.1780).

Grognet Fabrice, 2005. Objets de Musée, n'avezvous donc qu'une vie ?, Gradhiva 2, pp. 49-63.

Guillaud Laurent, Fanny Wonu Veys, Hélène Guiot et al., 2009. Tapa, étoffes cosmiques de l'Océanie, Cahors, musée de Cahors Henri-Martin.

Guiot Hélène (éd.), 2000. 'Uvea-Wallis, une île pêchée par les dieux, catalogue de l'exposition, Chartres, musée des beaux-arts de Chartres.

Guiot Hélène et Claude Stefani, 2002. Les objets océaniens, série polynésienne, vol. 1, Chartres, musée des beaux-arts de Chartres.

Hamy Ernest Théodore, 1880. Rapport de M. le Dr Hamy sur le développement et l'état actuel des collections ethnographiques, adressé au ministère de l'Instruction publique au nom d'une commission spéciale, Bulletin hebdomadaire de l'Association scientifique de France 2, 30, pp. 53-62.

JACQUEmin Sylviane, 1990-1991. Origine des collections océaniennes dans les musées parisiens : le musée du Louvre, Journal de la Société des Océanistes 90, pp. 47-52 (https://doi.org/10.3406/ jso.1990.2868).

—, 1991. Objets des mers du Sud: Histoire des collections océaniennes dans les musées et établis- sements parisiens, $\mathrm{XVIII}^{\mathrm{e}}-\mathrm{XX} \mathrm{X}^{\mathrm{e}}$ siècles, mémoire de recherche de l'école du Louvre.

—, 1994. Des objets océaniens rescapés de l'expédition d'Entrecasteaux (1791-1794), Journal de la Société des océanistes 99, pp. 207-208 (https://doi. org/10.3406/'so.1994.1939).

—, 1998. L'exploration des collections d'objets d'Océanie, La Lettre de l'OCIM 60, pp. 23-27.

—, 1999, La collection océanienne, in Dominique Vivant Denon, l'oil de Napoléon, cat. exp., Paris, Réunion des musées nationaux, pp. 433-436.

—, 2000. Objets du Pacifique dans les collections publiques françaises, in J. Kerchache (éd.), Sculptures. Afrique, Asie, Océanie, Amériques, Paris, RMN, pp. 34-43.

Jeudy-Ballini Monique, 1999. Dédommager le désir. Le prix de l'émotion en Nouvelle-Bretagne (Papouasie Nouvelle-Guinée), Terrain 32, pp.520 (DOI : https://doi.org/10.4000/terrain.2718).

KasarhÉrou Emmanuel, 2021. Du patrimoine kanak dispersé au patrimoine kanak partagé, in Carnets kanak. Voyage en inventaire de Roger Boulay, Paris, musée du quai Branly-Jacques Chirac, pp. 11-14.

Kaufmann Christian, Philippe Peltier et Markus Schindlbeck (éds), 2018. Journal de la Société des Océanistes 146 : Le Sepik: société et production matérielle (https://doi.org/10.4000/jso.7928).

Kerfant Céline, Magali melandri et Christophe moulherat, 2019. Pour une restitution d'un patrimoine (re)naissant: méthodes d'analyse et perspectives de l'imagerie numérique 3D sur un corpus d'éventails des îles Marquises, In Situ 39 (https://doi.org/10.4000/insitu.21725).

Kooljman Simon, 1972. Tapa in Polynesia, Honolulu, Hawaii Bishop Museum.

Langer Odette, 1996. Marie-Charlotte Laroche (1906-1996), Journal de la Société des Océanistes 103 , p. 319 (www.persee.fr/doc/jso_0300953x_1996_num_103_2_2004).

Laroche Marie-Charlotte, 1945. Pour un inventaire des collections océaniennes en France, Journal de la Société des Océanistes 1, pp. 51-57 (https://doi. org/10.3406/jso.1945.1485).

—, 1953a. Vers un inventaire des collections calédoniennes en France, Journal de la Société des Océanistes 9, p. 301 (www.persee.fr/doc/jso_0300953x_1953_num_9_9_2177).

—, 1953b. Collection d'objets calédoniens du Muséum de Toulouse, Journal de la Société des Océanistes 9, pp. 307-319 (https://doi.org/10.3406/ jso.1953.1781).

—,1953c. Collections calédoniennes du $\mathrm{Mu}-$ séum de Rouen, Journal de la Société des Océa- 
nistes 9, pp. 320-322 (https://doi.org/10.3406/ jso.1953.1782).

—, 1964. Notes sur un sarcophage en bois sculpté de Nouvelle-Calédonie conservé au Musée de Pithiviers, Journal de la Société des Océanistes 86-87, pp. 85-87 (https://doi.org/10.3406/ jso.1964.1906).

—, 1966. Collections océaniennes du Muséum d'Histoire naturelle du Havre, Journal de la Société des Océanistes 22 (22), pp. 77-93.

Lauriere Christine, 2017. L'épreuve du feu des futurs maitres de l'ethnologie, in André Delpuech et al., Les Années folles de l'ethnographie. Trocadéro 28-37, Paris, Muséum national d'histoire naturelle, pp. 405-447.

Lauriere Christine et Carine Peltier-Caroff, 2017. Liste des missions ethnologiques (19251939), in A. Delpuech et al., Les Années folles de l'ethnographie. Trocadéro 28-37, Paris, Muséum national d'histoire naturelle, pp. 915-919.

LAVONDES Anne, 1973. La culture matérielle en Polynésie d'après les collections archéologiques et ethnographiques du musée de Papeete, thèse de troisième cycle, Paris, université de la Sorbonne.

—, 1976. Collections polynésiennes du musée d'histoire naturelle de Cherbourg, Journal de la Société des Océanistes 32 (51-52), pp. 185-205 (https:// doi.org/10.3406/jso.1976.2742).

—, 1978. Les collections ethnographiques de l'Hôpital militaire de Brest, Objets et Mondes, La revue du musée de l'Homme 18, 1-2, pp. 91-95.

—, 1986, Les collections ethnographiques polynésiennes dans les musées français, Nouvelles des musées classés et contrôlés, pp. 5-26 (https://horizon.documentation.ird.fr/exl-doc/pleins_textes/ pleins_textes_5/b_fdi_18-19/23066.pdf).

—, 1990. Vitrine des objets océaniens: inventaire des collections du Muséum de Grenoble, Grenoble, Muséum d'histoire naturelle.

—, 1994. Les collections océaniennes du muséum de Perpignan, Annales du muséum d'histoire naturelle de Perpignan, pp. 3-12.

—, 2010. L'ethnologie et l'objet au "CFRE ", in Matières, manières et sociétés, hommage à Hélène Balfet, Aix-en-Provence, Publications de l'université de Provence, pp. 93-94.

LeBLic Isabelle (éd.), 2013. Journal de la Société des Océanistes 136-137 : La part «d'immatériel» dans la culture "matérielle» (https://doi.org/10.4000/ jso.6837).

LeClerc-Caffarel Stéphanie, 2008. Les collections fidjiennes conservées en France. L'exemple des collectes Dumont d'Urville, mémoire de recherche de $2^{\mathrm{e}}$ cycle en histoire de l'art appliquée aux collections, Paris, école du Louvre.
LenNard Frances et Andy mills, 2021. Material approaches to polynesian barkcloth, Leiden, Sidestone Press.

Merlin-Anglade Véronique, 1998. AquitaineOcéanie, Terres d'échanges, Bordeaux, Association des conservateurs des collections publiques de la région Aquitaine.

Neich Roger et Mike Pendergast, 1998. Traditional Tapa textiles of the Pacific, Londres, Thames $\&$ Hudson.

Patole-Edoumba Élise, 2009. Historiographie et histoire de l'art océanien, in Oissila Saaida et Laurick Zerbini (éds), La construction du discours colonial: l'empire français au XIX et XX' siècles, Paris, éditions Karthala, pp. 169-186.

Peltier Philippe, 1979. L'art océanien entre les deux guerres: expositions et vision occidentale, Journal de la Société des Océanistes 65, 35, pp. 271 282 (https://doi.org/10.3406/jso.1979.3016).

—, 1984. From Oceania, in W. Rubin (ed), "Primitivism" in $20^{\text {th }}$ century art: affinity of the tribal and the modern, New York, The Museum of Modern Art, pp. 99-121 (trad. franç., 1987, Paris, Flammarion).

Peltier Philippe et Magali MéLandri, 2012. Chronologie concernant les têtes tatouées et momifiées māori ou toi moko (aussi connues sous le terme de moko mokai), Journal de la Société des Océanistes 134, pp. 28-30 (https://doi.org/10.4000/ jso.6638).

Pequignot Amandine, 2016. Les muséums et les collections d'histoire naturelle: patrimonialisation et nouveaux défis pour le Xxi ${ }^{\mathrm{e}}$ siècle, In Situ. Revue des patrimoines 30 (http://insitu.revues. org/13871).

Pomian Krzystof, 2008. L'histoire des collections : audelà du temps des pionniers, Perspective 1, pp. 5-7.

—, 2021. Le musée, une histoire mondiale. I. Du trésor au musée, Paris, Gallimard.

Poulpiquet Anne-Claire de, 2012. Souvenir d'un tour du monde en écorce battue : un kapa hawaiien du XIX ${ }^{e}$ siècle (musée du quai Branly, Paris). Recherche sur les interactions entre le support de l'œuvre et son décor. Étude comparative d'adhésifs pour la consolidation de zones fragmentaires, mémoire de fin d'études, diplôme de restaurateur du patrimoine, INP.

Sarasin Fritz, 1929. Ethnologie der Neu-Caledonier und Loyalty-Insulaner, Berlin, C.W. Kreidel's Verlag (trad. franç. Raymond Ammann, Bernard Gasser et Christian Kaufmann, 2009, Paris, Ibis Press).

SARR Felwine et Bénédicte SAvoy, 2018. Rapport $\mathrm{n}^{\circ}$ 2018-26 sur la restitution du patrimoine africain. Vers une nouvelle éthique relationnelle.

Schnapper Alain, 1997. Les cabinets de curiosités, in Collectif, Océanie : La découverte du paradis : 
curieux, navigateurs et savants, Lille, Association des conservateurs des musées du Nord-Pas-deCalais, Somogy Éditions d'Art, pp. 19-22.

Sibeud Emannuelle, 2004. Marcel Mauss : «Projet de présentation d'un bureau d'ethnologie " (1913), Revue d'histoire des sciences humaines 1 (1), pp. 105-115 (https://doi.org/10.3917/ rhsh.010.0105).

Specht Jim and Lissant Bolton, 2005. Pacific islands' artefact collections: the UNESCO inventory project, Journal of Museum Ethnography $17: \mathrm{Pa}-$ cific Ethnography, Politics and Museums, pp. 58-74 (https://www.jstor.org/stable/40793769).

Speiser Félix, 1923. Ethnographische Materialien aus den Neuen Hebriden und den Banks-Inseln, Berlin,
C.W. Kreidel (trad. anglaise, 1996. Ethnology of Vanuatu: an early twentieth century study, Honolulu, University of Hawai'i Press).

Steinen Karl von den, 1925-1928. Die Marquesaner und ihre Kunst: Studien über die Entwicklung primitiver Südseeornamentik nach eigenem Reiseergebnissen und dem Material der Museum, Berlin, D. Reimer (E. Vohsen), 3 vol. (rééd. en français, 2005-2008. Les Marquisiens et leur art, Papeete, musée de Tahiti et des îles, Le Motu, 3 vol.).

Thomas Nicholas, Julie Adams, Billie LYTHberg, Maia Nuku \& Amiria Salmond, 2016. Artefacts of encounter: Cook's voyages, colonial collecting and museum histories, Honolulu, University of $\mathrm{Ha}$ waii i Press. 